University of Nebraska - Lincoln

DigitalCommons@University of Nebraska - Lincoln

July 1984

\title{
Modulated piezoreflectivity and the photoelasticity of molecular crystals
}

T. Luty

University of Nebraska - Lincoln

Craig J. Eckhardt

University of Nebraska - Lincoln, ceckhardt1@unl.edu

Follow this and additional works at: https://digitalcommons.unl.edu/chemistryeckhardt

Part of the Chemistry Commons

Luty, T. and Eckhardt, Craig J., "Modulated piezoreflectivity and the photoelasticity of molecular crystals" (1984). Craig J. Eckhardt Publications. 31.

https://digitalcommons.unl.edu/chemistryeckhardt/31

This Article is brought to you for free and open access by the Published Research - Department of Chemistry at DigitalCommons@University of Nebraska - Lincoln. It has been accepted for inclusion in Craig J. Eckhardt Publications by an authorized administrator of DigitalCommons@University of Nebraska - Lincoln. 


\title{
Modulated piezoreflectivity and the photoelasticity of molecular crystals ${ }^{a)}$
}

\author{
T. Luty ${ }^{\text {b) }}$ and C. J. Eckhardt \\ Department of Chemistry, University of Nebraska-Lincoln, Lincoln, Nebraska 68588-0304
}

(Received 1 February 1984; accepted 29 March 1984)

\begin{abstract}
Analysis of the optical response of a molecular crystal undergoing an external, periodic modulation is presented in terms of classical dipole theory. The case of modulated strain is treated in particular and identification of such strain with acoustic phonons with $q=0$ permits use of lattice dynamical theory to elucidate the acoustic phonon-exciton interaction. It is shown that extrema in the piezomodulated reflectivity (PMR) locate transverse and longitudinal excitonpolariton frequencies under specific experimental conditions. Model calculations demonstrate the great difference in the PMR for strong and weakly coupled systems. Relation of the PMR intensity to the elasto-optic coefficients and the storage of mechanical energy by the crystal permits extension of the technique to the study of such phenomena as internal strains and phase transitions where the partition of the mechanical energy among the various crystal excitations is of interest.
\end{abstract}

\section{INTRODUCTION}

The use of external perturbations to modulate the optical response of crystals has been widely exploited. ${ }^{1}$ Modulations which have some symmetry interactive character where the perturbation is tensorial in nature can be of particular utility. A potentially useful perturbation is strain (stress) wherein strains of specific symmetry are induced by application of a known stress to a crystal. If this applied stress is oscillatory, a modulation of the optical response, say the reflectivity, can be generated which can be detected with great sensitivity.

Previous experimental studies of the electronic spectra of molecular crystals by piezomodulated reflectivity yielded experimental and theoretical results which indicated great possibilities of the approach for the elucidation of the excitations of these solids. ${ }^{2-5}$ This spectroscopic technique has been employed in the study of metals and semiconductors but the difficulty in defining a general dielectric function for these materials severely inhibited further development.

The application to molecular crystals has led to discovery of a startling correspondence of the structure observed in the piezomodulation spectra observed at $300 \mathrm{~K}$ to spectra obtained by static or unmodulated spectroscopy at temperatures near $4 \mathrm{~K}^{3}$ The most intriguing example was found in the piezomodulation spectra of anthracene. ${ }^{6}$ Excellent correspondence was observed to directly measured absorption spectra when the ambient temperature piezomodulation spectra were shifted as a whole to the red to compensate for the bathochromic shift of the crystal absorption spectra upon cooling. Within the resolution of the experiments, peaks in the piezoreflection spectra were in agreement with the observed low temperature spectra. It must be stressed that the equivalent ambient temperature static measurement shows only the broadest features for anthracene.

Extension of the modulated piezoreflection spectroscopy to molecular crystals exhibiting quasimetallic reflection bands which are extremely resistant to sharpening at low

\footnotetext{
a) Supported by the NSF Solid State Chemistry Program through Grant DMR 79-08759.

b) Permanent address: Institute of Organic and Physical Chemistry, Technical University of Wroclaw, Wroclaw, Poland.
}

temperatures also revealed an unexpected richness of structure. ${ }^{5}$ In fact, most of this structure was observed in the socalled multiparticle region which had thereunto proven entirely resistant to efforts to enhance its structure. The salient features of these data were the appearance of distinct extrema at the high energy side of the band, where longitudinal polariton modes were expected. The fact that this structure was invariant with respect to the direction along which the spectra were obtained indicated that the piezomodulation spectra were locating the $\mathbf{k}=0$ polariton mode.

A model calculation based upon a two oscillator dielectric function reproduced the piezomodulated reflection spectra well. ${ }^{2}$ A clear correlation of the minima of the refiection band to the longitudinal frequencies was obtained. However, the detailed nature of the model dielectric function prevented a straightforward analysis of the piezomodulated reflection spectra and an unambiguous identification of the longitudinal modes with piezoreflection structure.

A more microscopic treatment is required which clarifies the nature of the piezomodulated reflection spectra and modulation spectroscopies in general. To accomplish this, the influence of the modulation parameter on the dielectric response must be formulated in general. In the case of the strain modulation, the identification of the strain with acoustic phonons is fruitful and the subsequent coupling to excitons can be then developed. This approach shows that the piezomodulation spectroscopy is a selective spectroscopy where acoustic phonons of the same symmetry are activated and involved in the coupling mechanisms of excitons. Consideration of these basic crystal excitations permits a straightforward analysis of the piezomodulated reflection spectra.

At visible light frequencies and higher, the optical properties of molecular crystals are determined by excitons which are the collective excitations due to intermolecular interactions. In the situation where acoustic phonons are involved it is useful to recognize that the van der Waals forces which largely determine the nature of these phonons are themselves determined by the exciton states. ${ }^{7}$ The excitons are related to the free molecule transitions. Thus, the concept of the exciton, while allowing a simplified model for 
the understanding of the optical properties of a crystal, does not itself offer a complete explanation of the complexities of a real system.

The low energy excitations, phonons, make the picture more complicated. Exciton theories ${ }^{8}$ describing excitonphonon coupling do so by introduction of damping which is quantitatively difficult to describe. Namely, the low energy phonons act as a channel for electronic energy decay and a damping constant is used as the easiest way to express the phonons' role in exciton spectra. Moreover, by forming multiparticle states, phonons are seen as a background for excitons. To learn more about exciton-phonon coupling in molecular crystals, a selective spectroscopy is required which will be able to detect a change in exciton spectra caused by particular phonon modes. It is the aim of this paper to show that piezomodulation spectroscopy, and in particular piezomodulated reflection spectroscopy of molecular crystals, is an effective selective method for study of the excitonphonon coupling. By applying modulated stress to a crystal, corresponding components of the strain tensor are produced. The strains can be viewed as the macroscopic correspondence of acoustic phonons with the frequency of the modulation. Alternatively, the modulated stress produces acoustic phonons whose amplitudes depend upon elastic properties of the crystal. To assure conditions appropriate for the propagation of an acoustic wave in the crystal, one has to provide a necessary balance between homogeneous deformation and internal strains. ${ }^{9}$ Synchronously measured reflectivity gives a response of electronic excitations to the particular stimulated phonons. Since the reflectivity of a crystal is determined by coupled exciton-photon modes, the piezomodulated reflection spectroscopy gives information about exciton-phonon as well as polariton-phonon coupling.

The coupling between electronic excitations and phonons has an effect on energies of both types of elementary excitations. The electronic excitations which mediate intermolecular interactions are taken into account in microscopic lattice dynamical theory. In the theory, ${ }^{10}$ the key quantity is the static dielectric function and many fruitful models have been developed to formulate that function in tractable form. ${ }^{11,12}$ In molecular crystals where the tight binding approximation is exploited, one can use some ideas from the theory of lattice dynamics to make the dielectric function in the crystals of a more general form. ${ }^{13}$

Since the poles of the high frequency dielectric function correspond to exciton energies in the crystal, the theoretical development for piezomodulated reflection spectroscopy will be to find the influence of low frequency acoustic phonons on the high frequency dielectric function. The problem is just the opposite to that found in lattice dynamical theory where the influence of the low frequency dielectric function on phonon energies is sought.

In terms of macroscopic quantities where the dielectric function is replaced by a dielectric constant tensor and the acoustic phonons by strains, the problem formulated will be answered by consideration of the elasto-optic tensor. ${ }^{14}$ However, since the dielectric constant has a complicated frequency dependence, a similar dependence will be followed by the elasto-optic constants. The dispersion is especially complicated in the region of exciton energies in molecular crystals. This is one of the reasons why a simple phenomenological interpretation of piezomodulated reflectivity using Seraphin coefficients ${ }^{1}$ is not appropriate for molecular crystals. For the same reason it is preferred to formulate the piezomodulated reflectivity response in terms of molecular properties in order to better understand dispersion of the elasto-optic coefficients in the excition energy region. From the basic knowledge of exciton-phonon coupling which can be obtained from the experiment, the information on the dispersion of the elasto-optic tensor can be helpful for studying enhanced Brillouin scattering from molecular solids.

The next two sections separately consider the dielectric function of the molecular crystal and the strain viewed as acoustic phonons due to the modulated stress. The main result will be derived in Sec. IV where the piezomodulated reflectivity response for a molecular crystal will be calculated and expressed in terms of molecular properties and intermolecular interactions. This will lead to the problem of elasto-optic coefficients and their dispersion. In Sec. V expressions for the resonances and intensities of the modulated piezoreflection spectra are obtained. Finally, phenomena are mentioned which can complicate the outlined description and in conclusion possible applications of the method as well as results obtained from model calculations are discussed.

\section{DIELECTRIC FUNCTIONS AND EXCITONS}

Since 1964 when G. D. Mahan published his paper on Davydov splittings in anthracene, ${ }^{15}$ the classical dipole theory has been recognized as the simplest approach for finding exact solutions of the exciton states in molecular crystals. In the theory, this many body problem is solved by the dielectric function which has a particularly simple form for molecular crystals. When the tight binding approximation is used, the dielectric function is expressed in terms of free molecule polarizabilities and Coulomb tensors which represent coupling between point dipoles. In this version the dipole theory oversimplifies exciton interactions and this is why it is often thought that the theory fails for so-called weak transitions. ${ }^{8}$ Site shifts and nondipolar exciton interactions must be included in any refinement of this theory which, in particular, means a refinement of the dielectric function.

Consider first the simplest formulation of the dielectric function for a molecular crystal with $Z$ molecules in the primitive unit cell. The free molecule polarizability tensor is defined as ${ }^{16}$

$$
4 \pi \boldsymbol{\alpha}_{k}^{0}(\omega)=\sum_{n} \frac{\omega_{0}^{2} f_{k}^{n}}{\omega_{n}^{2}-\omega^{2}} \mathbf{d}_{k}^{n} \otimes \mathbf{d}_{k}^{n}
$$

and the Fourier transform of point dipole-dipole interactions

$\mathrm{T}_{k k^{\prime}}(\mathbf{q})=\frac{\boldsymbol{V}}{4 \pi} \sum_{l} \frac{\exp \left(i \mathbf{q} \cdot \mathbf{R}_{k, l, k^{\prime}}\right)}{\boldsymbol{R}_{k, l, k^{\prime}}^{3}}\left[\mathbf{1}-\frac{3 \mathbf{R}_{k, l, k^{\prime}} \otimes \mathbf{R}_{k, l, k^{\prime}}}{\boldsymbol{R}_{k, l, k^{\prime}}^{2}}\right]$

with $\mathbf{R}_{k, l, k^{\prime}}=\mathbf{R}_{0 k}-\mathbf{R}_{l k^{\prime}}$ and $V$ the volume of the unit cell. The dipole theory gives the equation for the polarization response $\mathbf{P}(\mathbf{q}, \omega)$ of wave vector $q$ for an applied external field $\mathbf{E}(\mathbf{q}, \omega)$ of frequency $\omega$ : 
$\mathbf{P}(\mathbf{q}, \omega)=\alpha^{0}(\omega) \cdot\left[1+4 \pi T(\mathbf{q}) \cdot \alpha^{0}(\omega)\right]^{-1} \cdot \mathbf{E}(\mathbf{q}, \omega)$.

The unit transition dipole for the $n$th transition $d^{n}$ has an associated oscillator strength $f^{n}$ and resonance frequency $\omega_{n}$. The plasma frequency is $\omega_{0}$. The coordinate vectors $\mathbf{R}$ locate the $k$ th molecule in the $l$ th unit cell. The equation defines a $3 Z \times 3 Z$ dielectric susceptibility matrix ${ }^{17}$

$$
\chi^{0}(q, \omega)=\alpha^{0}(\omega)\left[1+4 \pi T(q) \cdot \alpha^{0}(\omega)\right]^{-1}
$$

and from the definition of the dielectric function ${ }^{12}$ follows the dipolar dielectric function:

$$
\varepsilon^{0}(\mathbf{q}, \omega)=1+4 \pi T(\mathbf{q}) \cdot \alpha^{0}(\omega) .
$$

The exciton energies are determined for zero applied field and are the poles of the dielectric susceptibility matrix. The exciton dispersion equation becomes

$$
\operatorname{det}\left|\varepsilon^{0}(\mathbf{q}, \omega)\right|=0 \text {. }
$$

It is worth noting that the van der Waals binding energy of a crystal can be written as ${ }^{7}$

$$
V_{d}=\frac{\hbar}{4 \pi N i} \sum_{\mathbf{q}} \oint_{c} \ln \left\{\operatorname{det}\left|\varepsilon^{0}(\mathbf{q}, \omega)\right|\right\} d \omega
$$

which expresses the difference between the zero point energies of the excitons and free molecule excitations.

The exciton eigenproblem formulated above corresponds to the tight binding approximation which completely neglects all short-range, nondipolar coupling between molecular excitations. This is the reason why the theory does not work well for the weak coupling limit when excitations are localized and short range effects are more important than very weak dipolar coupling. The effect seems to be important for the strong coupling limit as well. Therefore, one has to look for a more general form of the susceptibility and dielectric function. As mentioned in the introduction, very fruitful models have been developed in the theory of lattice dynamics. Based on these; the concept of effective and nonlocal polarizabilities to describe short range contributions to the dielectric response has been developed recently. ${ }^{13}$ This provides a phenomenological but suitable generalization of the dielectric function. The nondipolar interactions have various effects on the response of the polarizability-they replace the free molecule response $\boldsymbol{\alpha}_{k}^{0}(\omega)$ by an effective polarizability $\alpha_{k}(\omega)$ and introduce nonlocal polarizabilities $\gamma_{k k^{\prime}}(\mathbf{q}, \omega)$. Thus,

$$
\boldsymbol{\alpha}_{k k^{\prime}}(\mathbf{q}, \omega)=\boldsymbol{\alpha}_{k}(\omega) \delta_{k k^{\prime}}+\gamma_{k k^{\prime}}(\mathbf{q}, \omega)
$$

now replaces the free molecule polarizabilities. Explicit expressions for these components represented by a nondipolar interaction tensor can be found elsewhere. ${ }^{13}$ The effective polarizability $\boldsymbol{\alpha}_{k}(\omega)$ can still be seen as a molecular or local response of the molecule at site $k$ determined by excitation energies which include mean crystal field effects through the gas to crystal shift term, $D$. When the field can be approximated as isotropic, then ${ }^{13}$

$$
4 \pi \boldsymbol{\alpha}_{k}(\omega)=\sum_{n} \frac{\omega_{0}^{2} f_{k}^{n}}{\Omega^{2}-\omega^{2}} \mathbf{d}_{k}^{n} \otimes \mathbf{d}_{k}^{n} .
$$

The nonlocal polarizabilities arise from exchange-overlap interactions between molecules. Being no longer local, the polarizability acquires a dependence on wave vector

$$
\gamma_{k k^{\prime}}(\mathbf{q}, \omega)=\sum_{l^{\prime}} \gamma_{0 k, l^{\prime} k^{\prime}}(\omega) \exp \left(i q \cdot \mathbf{R}_{k, l^{\prime}, k^{\prime}}\right)
$$

This contribution to the polarizability implies that the polarization at a given site $(0 k)$ could involve the excited states associated with a different site $\left(l^{\prime} k^{\prime}\right) .{ }^{12}$ It should be clear that the phenomenologically introduced nonlocal polarizability will play an important role only if the electron orbitals are highly delocalized such as in semiconductors, but this is also true for some molecular solids. The similarity of this concept in the dielectric theory of semiconductors to that of charge transfer transitions in molecular crystals is obvious but rigorous treatment is required to include the contribution of these to the dielectric function of molecular solids. ${ }^{13}$

Equations (3)-(5) can be rewritten with $\boldsymbol{\alpha}_{k}^{0}(\omega)$ replaced by $\boldsymbol{\alpha}_{k}(\omega)$ of Eq. (8) and

$$
\varepsilon(\mathbf{q}, \omega)=1+4 \pi T(\mathbf{q}) \cdot \boldsymbol{\alpha}(\mathbf{q}, \omega)
$$

which is the required generalization of the dielectric function. The exciton energies are obtained from

$$
\operatorname{det}|\varepsilon(\mathbf{q}, \omega)|=0 \text {. }
$$

When the external field $\mathbf{E}(\mathbf{q}, \omega)$ in Eq. (3) is nonzero, excitonphoton interactions lead to formation of polaritons. For a transverse field the polariton dispersion equation is

$$
\operatorname{det}\left|\boldsymbol{\epsilon}(\mathbf{q}, \omega)-n^{2}(\mathbf{q}, \omega)[\mathbf{1}-\hat{\mathbf{q}} \otimes \hat{\mathbf{q}}]\right|=\mathbf{0},
$$

where $n(\mathbf{q}, \omega)=c q / \omega$ and $\hat{\mathbf{q}}$ is the unit vector of the wave vector. $\mathbf{\epsilon}(\mathbf{q}, \omega)$ is the macroscopic dielectric constant tensor which when expressed in terms of the polarizabilities becomes $^{18}$

$$
\begin{aligned}
\mathbf{\epsilon}(\mathbf{q}, \omega) & =\mathbf{1}+\frac{1}{Z^{2}} \sum_{k k^{\prime}}\left[\frac{1}{4 \pi} \boldsymbol{\alpha}^{-1}(\mathbf{q}, \omega)+\mathrm{T}(\mathbf{q})\right]_{k k^{\prime}}^{-1} \\
& =\mathbf{1}+\frac{1}{Z^{2}} \sum_{k k^{\prime}}\left[4 \pi \boldsymbol{\alpha}(\mathbf{q}, \omega) \cdot \varepsilon^{-1}(\mathbf{q}, \omega)\right]_{k k^{\prime}}^{-1} .
\end{aligned}
$$

It follows from the above relation between the dielectric constant tensor and the dielectric function that the exciton frequencies correspond to the poles of the dielectric constant tensor while polariton frequencies correspond to zeros of the tensor. However, actually measured exciton and polariton frequencies correspond to the poles and zeros, respectively, of the right-hand side of Fresnel's equation

$$
n^{2}(\mathbf{q}, \omega)=\left\{\mathbf{\epsilon}^{-1}(\mathbf{q}, \omega):[1-\hat{\mathbf{q}} \otimes \hat{\mathbf{q}}]\right\}^{-1} .
$$

This equation is quite general and with Eq. (14) expresses the refractive index of a crystal measured with light of frequency $\omega$ and wave vector $\mathbf{q}$. For light polarized along $\hat{\mathbf{x}}^{20}$ :

$$
n^{2}(\mathbf{q}, \omega)=\left\{\hat{\mathbf{x}} \cdot \boldsymbol{\epsilon}^{-1}(\mathbf{q}, \omega) \cdot \hat{\mathbf{x}}\right\}^{-1} .
$$

The above description makes it possible, in principle, to calculate the optical response of a molecular crystal from molecular properties and dipolar coupling. It looks especially simple when free molecule polarizabilities are used. In practice, it does not work, ${ }^{21}$ even for the anthracene crystal, where this approximation gives excellent Davydov splittings. The reason is that short-range exchange interactions play a substantial role in determining optical properties and this is why the concept of effective molecular polarizabilities as well as the nonlocal polarizabilities in some cases have to be taken into account. This would suggest that the optical properties of molecular crystals are determined by excitations which are collective not only because of dipolar coupling but also because of short-range coupling between mo- 
lecular excitations. If so, these effects should not be overlooked, at least qualitatively, in the spectroscopy of molecular crystals.

\section{STRESS, STRAIN AND ACOUSTIC PHONONS}

Equation (13) determines the propagation of polaritons in the crystal. It shows that if the dielectric constant tensor for a given wave vector and frequency is known, the velocity of waves in the crystal can be determined and the waves characterized by eigenvectors of the equation which are unit vectors of the polarization waves.

The analogous equation which governs the propagation of elastic waves in the crystal is the Christoffel equation',

$$
\operatorname{det}\left|\boldsymbol{\Gamma}-\mathbf{1} \rho v^{2}\right|=0 \text {, }
$$

where $\Gamma_{\alpha \beta}=C_{\alpha \gamma \beta \delta} \hat{q}_{\gamma} \hat{q}_{\delta}, \hat{q}_{\gamma}$ and $\hat{q}_{\delta}$ are the direction cosines of the propagation vector, the $C_{\alpha \gamma \beta \delta}$ are the stiffnesses, $\rho$ is the density of the crystal, and $v$ is the elastic wave velocity. It is important to recall lattice dynamical analysis of the stiffnesses in molecular crystals in order to make clear what kind of molecular motions are involved in the elastic response of the crystal when a stress is applied.

The elastic properties of nonpiezoelectric molecular crystals are conveniently described when the limit of long waves is taken for the secular equation describing phonon dispersion:

$$
\left[G(\mathbf{q})-m \omega^{2}(\mathbf{q})\right] \cdot V(\mathbf{q})=0 .
$$

Here, $G(q)$ is a $6 Z \times 6 Z$ harmonic lattice dynamical matrix composed of translational and rotational submatrices $\mathrm{G}\left(\mathrm{q} \cdot k k^{\prime}\right)$, where $k$ is the index of the sublattice. $\mathbf{V}(\mathbf{q})=\{\mathbf{u}(\mathbf{q}, k), \boldsymbol{\theta}(\mathbf{q}, k)\}$ represents the amplitudes of translational and rotational displacements of the molecules and $m$ is determined by the mass, $\mu$, and moment of inertia tensor. The perturbation method gives the following dynamical equation for acoustic phonons $j=1,2,3^{22}$ :

$$
\left(\begin{array}{cc}
\mathrm{G}_{\gamma \delta}^{(2)} \hat{q}_{\gamma} \hat{q}_{\delta}-\mathbf{1} v^{2}(q j) \mu & -i \mathrm{G}_{\gamma}^{+(1)} \hat{q}_{\gamma} \\
i \mathrm{G}_{\delta}^{(1)} \hat{q}_{\delta} & \mathrm{G}^{(0)}
\end{array}\right)\left(\begin{array}{c}
\mathbf{u}(\mathbf{q} j) \\
\mathbf{W}^{(1)}(\mathbf{q} j)
\end{array}\right)=0,
$$

where $\mathrm{G}^{(i)}$ stands for the $i$ th derivative of the dynamical matrix with respect to the wave vector in the limit $q \rightarrow 0 . \mathbf{u}(\mathbf{q} j)$ is the amplitude of the translational displacement of the primitive unit cell and $W^{(1)}(\mathbf{q} j)$ is the amplitude of intersublattice displacements which accompany the propagation of the given acoustic phonon $(\mathbf{q} j)$. $\mathbf{G}^{(0)}$ is determined by frequencies of all optical phonons at $\mathbf{q}=0$ and its inverse can be identified as the optical phonon susceptibility. By introduction of effective elastic constants

$$
C_{\alpha \gamma, \beta \delta}=\frac{1}{V}\left\{G_{\alpha \beta, \gamma \delta}^{(2)}-G_{\alpha \gamma, i}^{+}\left[G^{(0)}\right]_{i j}^{-1} G_{j, \beta \delta}^{(1)}\right\}
$$

it follows that

$$
\left(\boldsymbol{\Gamma}-1 \rho v^{2}\right) \cdot \mathbf{u}=0 .
$$

Eigenvalues of the matrix correspond to velocities of the three acoustic phonons (qi) propagating in the direction q. Eigenvectors of the matrix $u(q)$ give the translational displacement of molecules within the cell. These internal strains are given by

$$
\mathbf{W}^{(1)}(\mathbf{q} j)=\boldsymbol{\gamma} \cdot \mathbf{e}(\mathbf{q} j),
$$

where the product $q \otimes u(q j)$ in the limit of $q \rightarrow 0$ may be identified as the strain tensor $e(\mathbf{q} j)$ produced by the acoustic phonon $(\mathbf{q} j)$. The third rank tensor $\gamma$ is expressed in terms of $\left[G^{(0)}\right]^{-1}$ and $G^{(1)}$ matrices. ${ }^{22}$

These equations can be useful for the piezomodulation spectroscopy of molecular crystals where application of a low frequency fluctuating force by means of a piezoelectric device in adhesive contact with the crystal in a particular direction $q$ normal to a crystal face stimulates elastic waves. Knowledge of the elastic constant tensor of the crystal allows determination of the velocities and eigenvectors $\mathbf{u}(\mathbf{q} j)$ of three acoustic phonons. Then from the definition

$$
e_{\alpha \beta}(q j)=\lim _{\mathbf{q} \rightarrow 0} q_{\alpha} u_{\beta}(\mathbf{q} j)
$$

the strain tensor components produced by the phonons are determined. Finally, Eq. (22) which provides a necessary balance between homogeneous strain and internal molecular displacements gives internal strains. Equation (22) has to always be taken into account to assure conditions for the propagation of acoustic phonons in the crystal under fluctuating force. The amplitude of the strain is determined by the amplitude of the applied force assuming that there is no attenuation in the crystal. Because linear dispersion of the acoustic phonons is assumed, strain produced by an applied fluctuating force, and thus by acoustic phonons, will be independent of the frequency of the fluctuation but will be dependent on its amplitude. This will not be the case for crystals undergoing ferroelastic phase transitions at temperatures close to the transition temperature. Here, anomalous dispersion of the acoustic phonons will make the produced strain frequency dependent.

\section{EXCITON-ACOUSTIC PHONON COUPLING AND ELASTO-OPTIC PROPERTIES}

Although there have been treatments of the interactions of excitons with static strains, ${ }^{23}$ there are few attempts to be found in the literature ${ }^{24}$ which deal with the excitonacoustic phonon problem in relation to molecular properties. Here, without loss of generality, the dipole theory is employed to describe the exciton system and the acoustic phonons are represented by the strains which they produce in the lattice.

Equation (3) of Sec. II may be arranged to

$\left[\boldsymbol{\alpha}^{-1}(\mathbf{q}, \omega)+4 \pi T(\mathbf{q})\right] \cdot \mathbf{P}(\mathbf{q}, \omega) \equiv \chi^{-1}(\mathbf{q}, \omega) \cdot \mathbf{P}(\mathbf{q}, \omega)=\mathbf{E}(\mathbf{q}, \omega)$,

where the generalized polarizability $\boldsymbol{\alpha}(\mathbf{q}, \omega)$ has been used instead of the free molecule quantity. For zero applied field, the eigenvalues of $\chi^{-1}(q, \omega)$ correspond to exciton frequencies $\omega_{\lambda}(\mathbf{q})$ and from the orthogonality relation between eigenvectors $\mathbf{P}_{\lambda}(\mathbf{q})$ we can write

$$
\chi(\mathbf{q}, \omega)=\sum_{\lambda} \frac{\mathbf{P}_{\lambda}(\mathbf{q}) \otimes \mathbf{P}_{\lambda}(-\mathbf{q})}{\omega_{\lambda}^{2}(q)-\omega^{2}} \equiv \sum_{\lambda} \chi_{\lambda}(\mathbf{q}, \omega) .
$$

The crystal energy associated with the excitons is

$$
V_{\text {ex }}=\frac{1}{2} \sum_{q \lambda} \omega_{\lambda}^{2}(\mathbf{q}) P_{\lambda}(\mathbf{q}) P_{\lambda}(-\mathbf{q})
$$

where, as a consequence of the dipole theory used to describe 
the exciton system, polarization vectors $\mathbf{P}_{\lambda}(\mathbf{q})$ are used as normal coordinates of the excitons. Since the dipole theory gives an extremely transparent physical picture of the system, it shall be exploited further for the system of excitons under perturbation produced by acoustic phonons. In this theory, excitations which are poles of the polarizability tensor $\boldsymbol{\alpha}(\mathbf{q}, \omega)$ and are characterized by transition dipoles are coupled through dipole-dipole interactions represented by the $T(q)$ term. In effect, excitons, are formed which are characterized by an orthogonal set of polarization vectors $\mathbf{P}_{\lambda}(\mathbf{q})$. However, when a perturbation to the free exciton system is applied, the vectors $\mathbf{P}_{\lambda}(\mathbf{q})$ are no longer orthogonal and the excitons $(q, \lambda)$ will interact with each other and respond not only to the external field $\mathbf{E}(\mathbf{q}, \omega)$ but also to a field due to the perturbation. In particular, a strain e associated with acoustic phonons may cause a field given by other excitons. This can be written as

$\mathbf{P}_{\lambda}(\mathbf{q}, \omega)=\chi_{\lambda}(\mathbf{q}, \omega)\left[\mathbf{E}(\mathbf{q}, \omega)-\sum_{\lambda^{\prime}}\left(\mathbf{R}_{\lambda \lambda^{\prime}}(\mathbf{q}, \omega): \mathbf{e}\right) \cdot \mathbf{P}_{\lambda^{\prime}}(\mathbf{q}, \omega)\right]$,

where $R_{\lambda \lambda}(\mathbf{q}, \omega)$ is, in general, a fourth rank tensor describing exciton-acoustic phonon coupling. Equation (27) can be rearranged to give the susceptibility for the coupled excitonphonon system

$$
\sum_{\lambda \lambda^{\prime}}\left[\chi^{-1}(\mathbf{q}, \omega)+\mathrm{R}(\mathbf{q}, \omega): e\right]_{\lambda \lambda^{\prime}}^{-1}=X(\mathbf{q}, \omega, e) .
$$

Exactly the same result will be obtained with use of general susceptibilities within the time-dependent Hartree approximation. In the above equation $\chi(\mathbf{q}, \omega)$ is the single particle uncoupled susceptibility and R:e is the coupling. Similar to the exciton theory where molecular excitations are coupled by dipolar interactions, the excitons here are coupled by strain due to acoustic phonons. Symmetry rules for the coupling will be seen clearly from the contribution of the coupling to the crystal energy

$$
V_{\text {ex-ph }}=-\sum_{\lambda \lambda^{\prime}} \mathbf{P}_{\lambda}(\mathbf{q}) \cdot \mathrm{R}_{\lambda \lambda} \cdot(\mathbf{q}): \mathrm{e} \cdot \mathbf{P}_{\lambda} \cdot(-\mathbf{q})
$$

which has to be totally symmetric. The totally symmetric component of the strain tensor will couple excitons of the same symmetry while nonsymmetric strain components will couple excitons of differing symmetry.

The anthracene crystal serves as an example of this. For the $C_{2 h}^{5}$ space group, strain components $e_{11}, e_{22}, e_{33}$, and $e_{13}$ which are of $A_{g}$ symmetry and at $\mathrm{q}=0$ will couple excitons of $A_{u}$ or $B_{u}$ symmetries; components $e_{12}$ and $e_{23}$ of $B_{g}$ symmetry will couple excitons of $A_{u}$ with $B_{u}$ symmetries. So for $e_{12}$ or $e_{23}$ strains the ground electronic state of the crystal is no longer of $A_{g}$ but of $B_{g}$ symmetry. Due to the coupling between $A_{u}$ and $B_{u}$ excitons mediated by an acoustic phonon producing $e_{12}$ or $e_{23}$ strains, the elements $X_{12}(q=0, \omega, e)$ and $X_{23}(\mathbf{q}, \omega, \mathrm{e})$ of the dielectric susceptibility of anthracene crystal will be nonzero.

The eigenvalues of the $X^{-1}(q, \omega, e)$ correspond to new excitations formed in the crystal under strain. Corresponding eigenvectors $\mathbf{P}_{\phi}^{\prime}(\mathbf{q})$ form orthogonal sets and the coupled susceptibility can be represented as

$$
X(\mathbf{q}, \omega, e)=\sum_{\phi} \frac{\mathbf{P}_{\phi}(\mathbf{q}) \otimes \mathbf{P}_{\phi}(-\mathbf{q})}{\omega_{\phi}^{2}-\omega^{2}} .
$$

It is important to notice that the exciton-acoustic phonon matrix $R$ is much more general than a simple expansion of exciton energies in a power series with respect to strain. It accounts for the change in symmetry of excitons in the crystal under external perturbation. The secular equation for a crystal under strain which determines the exciton frequencies takes the form

$$
\left|\left(\chi^{-1}(\mathbf{q})+\mathbf{R}(\mathbf{q}): e\right)_{\alpha \beta}-\omega_{\phi}^{2}(\mathbf{q}) \delta_{\alpha \beta}\right|=0
$$

and that for the unstrained crystal is

$$
\left|\chi_{\alpha \beta}^{-1}(\mathbf{q})-\omega_{\lambda}^{2}(\mathbf{q}) \delta_{\alpha \beta}\right|=0 \text {. }
$$

Thus, the $R$ tensor can be related to a change in exciton frequencies,

$$
\left|(\mathbf{R}(\mathbf{q}): e)_{\alpha \beta}-\psi(\mathbf{q}) \delta_{\alpha \beta}\right|=0
$$

with

$$
\psi(\mathbf{q})=\omega_{\phi}^{2}(\mathbf{q})-\omega_{\lambda}^{2}(\mathbf{q}) \simeq 2 \omega_{\lambda}(\mathbf{q}) \Delta \omega(\mathbf{q}) .
$$

An exact expression for the elements of the coupling matrix can be deduced and expressed for simplicity in terms of the free molecule polarizability

$R(\mathbf{q}, \omega)=\left.\frac{\partial \chi^{-1}(\mathbf{q}, \omega)}{\partial e}\right|_{e=0}=\frac{\partial\left[\alpha^{0}(\omega)\right]^{-1}}{\partial \mathbf{W}}: \gamma+4 \pi \frac{\partial T(q)}{\partial e}$,

where $W$ is the internal strain vector related to the strain e by the tensor $\gamma$. This result corresponds to the case where molecules occupy sites with an inversion center and for such crystals the internal strain corresponds to rotational displacements of the molecules only. On the other hand, because the free molecule polarizability has been used, the external strain $e$ and the internal one $W$ act separately on $T(q)$ and $\boldsymbol{\alpha}^{0}(\omega)$, respectively.

Having obtained the dielectric susceptibility for the coupled exciton-acoustic phonon system, the elasto-optic tensor can be calculated. The derivative of $X(\omega, e)$ with respect to a homogeneous elastic strain $e$ is the same as the derivative of the dielectric constant tensor $\boldsymbol{\epsilon}(\omega, \mathrm{e})$. Then

$$
\frac{\partial X_{\alpha \beta}(\omega)}{\partial e_{\sigma \tau}} \equiv k_{\alpha \beta, \sigma \tau}(\omega)=-\frac{1}{4 \pi} \epsilon_{\alpha \gamma}(\omega) P_{\gamma \delta, \sigma \tau}(\omega) \epsilon_{\delta \beta}(\omega),
$$

where

$$
p_{\gamma \delta, \sigma \tau}(\omega)=\frac{\partial \epsilon_{\gamma \delta}^{-1}(\omega)}{\partial e_{\sigma \tau}}
$$

is the fourth-rank elasto-optic tensor. In these equations the frequency dependence of the tensor components is retained while assuming the wave vector $q=0$ as suitable for optical experiments. From Eq. (31) we have

$$
\mathrm{k}(\omega)=-\chi(\omega) \cdot \mathbf{R}(\omega) \cdot \chi(\omega)
$$

and

$\mathrm{p}(\omega)=-4 \pi\left[\chi^{-1}(\omega)+4 \pi 1\right]^{-1} \cdot \mathrm{R}(\omega) \cdot\left[\chi^{-1}(\omega)+4 \pi 1\right]^{-1}$

which show the relation between the microscopic excitonacoustic phonon coupling tensor $R(\omega)$ expressed in terms of molecular properties [Eq. (34)] and the macroscopic elastooptic coefficients. ${ }^{19}$

Equations (34) and (35) are generally valid over the entire range of frequencies of excitations taken into account in 
the susceptibility $\chi(\omega)$. The elasto-optic coefficients will have singularities corresponding to the poles of $\chi(\omega)$ which are exciton frequencies. It may be verified that the symmetry of the coupling tensor $R(\omega)$ is the same as the elasto-optic tensor p. The main difference between the two tensors lies not only in that $R(\omega)$ has microscopic meaning while that of $p$ is essentially macroscopic but also in that their dispersions differ significantly. While $p(\omega)$ will display some singularities, the coupling tensor $R(\omega)$ will be a rather smooth function of the frequency according to Eq. (31). For this reason, it is more appropriate for molecular crystals to define

$$
R(\omega)=\left\{R_{\alpha \beta, \sigma \tau}(\omega)\right\}
$$

as the photoelastic tensor. This form is most suited to solids whose optical properties are determined by Frenkel excitons. The main advantage of this new tensor is that it lends itself to microscopic interpretation. $R_{\alpha \beta, \sigma \tau}(\omega)$ can be seen as a measure of the coupling between excitons whose symmetries correspond to the transformation properties of vector components $V_{\alpha}$ and $V_{\beta}$ and are mediated by acoustic phonons which produce or are a result of $e_{\alpha r}$ strain in the crystal lattice.

A similar tensor can be defined with respect to the stress

$$
N_{\alpha \beta, \sigma \tau}(\omega)=\frac{\partial \chi_{\alpha \beta}^{-1}(\omega)}{\partial \sigma_{\sigma r}}
$$

and the relation between the $R$ and $N$ tensors is

$$
N=R \cdot C^{-1} \text {, }
$$

where $\mathrm{C}^{-1}$ is the compliance tensor.

Finally, note that the introduced tensor $R$ can be interpreted also as a deformation potential as evident from Eq. (29). However, when considering Frenkel excitons and thus most molecular solids, the deformation potential concept is not particularly useful and a microscopic interpretation of tensor $R$ is preferred.

\section{STRESS (STRAIN) MODULATED REFLECTIVITY OF NONPIEZOELECTRIC MOLECULAR CRYSTALS}

When a nonpiezoelectric molecular crystal is subject to a strain and incident light with wave vector $q$, there is no direct coupling between strain and the electric field of the light. Thus, only excitons in the strained crystal are coupled to the electric field and the new excitations are determined by the conditions

$$
\operatorname{det}\left|\boldsymbol{\epsilon}^{-1}(\mathbf{q}, \omega, \mathbf{e}) \cdot[\mathbf{1}-\hat{\mathbf{q}} \otimes \hat{\mathbf{q}}]\right|=0
$$

for excitons and

$$
\operatorname{det}\left|\left\{\boldsymbol{\epsilon}^{-1}(\mathbf{q}, \omega, \mathbf{e}) \cdot[\mathbf{1}-\hat{\mathbf{q}} \otimes \hat{\mathbf{q}}]\right\}^{-1}\right|=0
$$

for polaritons. The refractive index of the crystal measured with light of frequency $\omega$ and wave vector $q$ is

$$
n^{2}(\mathbf{q}, \omega, \mathbf{e})=\left\{\boldsymbol{\epsilon}^{-1}(\mathbf{q}, \omega, \mathrm{e}):[\mathbf{1}-\hat{\mathbf{q}} \otimes \hat{\mathbf{q}}]\right\}^{-1} .
$$

More specifically, for polarized light with $\mathbf{E}$ vector parallel to the unit vector $\hat{\mathbf{x}}$ :

$$
n^{2}(\mathbf{q}, \omega, \mathbf{e})=\left\{\boldsymbol{\epsilon}^{-1}(\mathbf{q}, \omega, \mathbf{e}):(\hat{\mathbf{x}} \otimes \hat{\mathbf{x}})\right\}^{-1} .
$$

For a statically deformed crystal, normal incidence specular reflectivity is given by

$$
\mathscr{R}(\mathbf{q}, \omega, \mathbf{e})=\left|\frac{n(\mathbf{q}, \omega, \mathrm{e})-1}{n(\mathbf{q}, \omega, \mathrm{e})+1}\right|^{2}
$$

with complex refractive index determined by Eq. (40) for a given direction of the $q$ vector with respect to an orthogonal crystal axial system. In experiments with modulated stress (strain) applied to the crystal, a difference between reflectivities $\mathscr{R}(\mathbf{q}, \omega,+e)$ and $\mathscr{R}(\mathbf{q}, \omega,-e)$ relative to the reflectivity for the undeformed crystal $\mathscr{R}(\mathbf{q}, \omega)$ is normally measured. This strain modulated response is

$$
2 \frac{\partial \ln \mathscr{R}(\mathbf{q}, \omega, \mathrm{e})}{\partial \mathrm{e}} \cdot \mathrm{e}=\zeta^{\prime}(\mathbf{q}, \omega) .
$$

It follows from Eq. (42) that

$$
\zeta^{\prime}(\mathbf{q}, \omega)=\frac{2}{n^{2}(\mathbf{q}, \omega)-1} \cdot \frac{\partial n(\mathbf{q}, \omega, \mathrm{e})}{\partial \mathrm{e}} \cdot \mathrm{e}
$$

which, when expressed in terms of the traditional elastooptic tensor $p$, takes the form

$$
\zeta^{\prime}(\mathbf{q}, \omega)=\frac{-n^{3}(\mathbf{q}, \omega)}{n^{2}(\mathbf{q}, \omega)-1} \cdot \mathbf{p}(\omega):[\mathbf{1}-\hat{\mathbf{q}} \otimes \hat{\mathbf{q}}]: \mathbf{e} .
$$

The refractive index term will produce singularities in the modulated reflectivity at exciton frequencies of the unstrained crystal which correspond to $n(\mathbf{q}, \omega) \rightarrow \infty$. However, as discussed previously, the elasto-optic tensor will also have some singularities and thus detailed analysis of the other terms in the expression is necessary. Additional consideration should be given to these singularities. Maxima and minima of the function $\xi^{\prime}(q, \omega)$ correspond to singularities for undamped oscillations. Since it is always possible and usually needed to introduce phenomenological damping, all tensors which are involved in this development are taken to be finite at every frequency. This permits inversion of the tensors such that valid expressions may be derived in the region of exciton polariton resonances.

To obtain resonances in the right-hand side of Eq. (45), the elasto-optic tensor is expressed in terms of the smoothly varying function $R(\omega)$ introduced previously

$$
\mathrm{P}:[1-\hat{\mathbf{q}} \otimes \hat{\mathbf{q}}]=4 \pi \epsilon^{-1} \cdot \chi \cdot \mathrm{R} \cdot \chi \cdot \epsilon^{-1} \cdot[1-\hat{\mathbf{q}} \otimes \hat{\mathbf{q}}],
$$

where frequency dependence has been dropped for simplicity. From this it is seen that the elasto-optic tensor will show singularities at both exciton $[\chi(\omega) \rightarrow \infty]$ and polariton $[\chi(\omega) \rightarrow 0]$ frequencies. However, whether these resonances will be observed depends upon experimental conditions defining the matrix $([\mathbf{1}-\hat{\mathbf{q}} \otimes \hat{\mathbf{q}}])$ which selects those points from $\mathrm{Eq}$. (45). Therefore, it is necessary to specify experimental conditions and then obtain expressions for the singularities.

Consider a monoclinic crystal and let the orthogonal crystal axes $a, b, c^{\prime}$ be parallel to the laboratory system $1,2,3$. Then in the laboratory system, the dielectric tensor is

$$
\epsilon=\left(\begin{array}{ccc}
\epsilon_{11} & 0 & \epsilon_{13} \\
0 & \epsilon_{22} & 0 \\
\epsilon_{13} & 0 & \epsilon_{33}
\end{array}\right)
$$

and its inverse

$$
\begin{aligned}
\epsilon^{-1}= & \epsilon_{22}\left(\epsilon_{11} \epsilon_{33}-\epsilon_{13}^{2}\right)^{-1} \\
& \times\left(\begin{array}{ccc}
\epsilon_{22} \epsilon_{33} & 0 & -\epsilon_{22} \epsilon_{13} \\
0 & \epsilon_{11} \epsilon_{33}-\epsilon_{13}^{2} & 0 \\
-\epsilon_{22} \epsilon_{13} & 0 & \epsilon_{11} \epsilon_{22}
\end{array}\right) .
\end{aligned}
$$


The frequency dependence has been dropped to simplify notation. For light normally incident upon the (001) face of the crystal, there are two cases of interest.

Case 1: $E$ is parallel to the $b$ axis. Here exciton frequencies are found from

$$
\epsilon_{22}^{-1}(\omega)=0 \rightarrow \chi_{22}(\omega) \rightarrow \infty
$$

and polariton frequencies from

$$
\epsilon_{22}(\omega)=0 \text {. }
$$

These excitations are called ordinary transverse excitons and ordinary longitudinal polaritons, respectively. With light polarized along $b$, the nonzero components of the elastooptic tensor are

$$
p_{22, \sigma \tau}(\omega)=4 \pi\left[\epsilon_{22}^{-1}(\omega)\right]^{2} \chi_{22}^{2}(\omega) R_{22, \sigma \tau}(\omega)
$$

which follows from Eq. (46). This demonstrates that the $p_{22, \sigma \tau}(\omega)$ elasto-optic coefficient will exhibit singularities at ordinary exciton as well as polariton frequencies. For nontotally symmetric components of the strain tensors $e_{12}$ and $e_{23}$, $R_{22,12}$ and $R_{22,23}$ will be zero and so corresponding components of the elasto-optic tensor will also vanish which is consistent with expectation for the monoclinic crystal. Furthermore, elasto-optic coefficients from Eq. (49) can be written as

$$
p_{22, \sigma \tau}(\omega)=\frac{1}{4 \pi}\left(\frac{n^{2}(\omega)-1}{n^{2}(\omega)}\right)^{2} R_{22, \sigma \tau}(\omega)
$$

and finally, the strain modulated reflectivity response for the $e_{\sigma \tau}$ component of the strain with incident light with $\mathbf{q} \| c^{\prime}$ and $\mathbf{E} \| b$ is

$$
\zeta(\omega)=\frac{1-n^{2}(\omega)}{4 \pi n(\omega)} \cdot R_{22, \sigma \tau} \cdot e_{\sigma \tau} .
$$

The result shows that the strain modulated reflectivity response is conveniently expressed in terms of the $R(\omega)$ tensor components which are smooth functions of frequency and, what is more important, have a well-defined microscopic meaning as coupling constants between excitons due to acoustic phonons producing a particular strain in the lattice. Thus, for an assumed geometry of the optical experiment there will be no response $\zeta(\omega)$ for nonsymmetric strains which is an advantage of this selective spectroscopy. Another advantage of the piezomodulation is that singularities in the $\zeta(\omega)$ function can be easily identified as corresponding to ordinary excitons and polaritons for the assumed geome- try. Experimentally observed spectra will be a convolution of the term $\left(n^{2}-1 / n\right)$ in which damping may be included and the smooth function of the frequency given by $R(\omega)$. The sign of a specific measured component of the tensor $R(\omega)$ can be determined by observing that for a positive sign the observed spectra should exhibit minima for transverse excitons.

Case 2: $E$ is parallel to the $a$ axis. Using Eq. (40) and the inverse dielectric constant, the expression for the refractive index is obtained

$$
n^{2}(\omega)=\epsilon_{11}(\omega)-\epsilon_{33}^{-1}(\omega) \epsilon_{13}^{2}(\omega)=\left[\epsilon^{-1}(\omega)\right]_{11}^{-1}
$$

and extraordinary exciton and polariton frequencies are determined from

$$
\epsilon_{33}(\omega)=0
$$

and

$$
\epsilon_{11}(\omega)-\epsilon_{33}^{-1}(\omega) \epsilon_{13}^{2}(\omega)=0,
$$

respectively. For the assumed geometry and polarization, the elasto-optic tensor component becomes

$p_{11, \sigma \tau}(\omega)=-4 \pi\left\{n^{-4}(\omega) k_{11, \sigma \tau}(\omega)+\left[\epsilon^{-1}(\omega)\right]_{13}^{2} k_{33, \sigma \tau}(\omega)\right\}$,

where from Eq. (34)

$$
k_{\alpha \alpha, \sigma \tau}(\omega)=-\chi_{\alpha \beta}(\omega) R_{\beta \gamma, \sigma \tau}(\omega) \chi_{\gamma \alpha}(\omega) .
$$

The dispersion of the $k(\omega)$ tensor can be analyzed when exact formulas for the susceptibility components $\chi_{\alpha \beta}(\omega)$ are ascertained. By defining the angles $\phi_{\lambda}$ with respect to the axis $a \| 1$ to indicate polarization vector directions of the $\lambda$ th exciton in the $a c$ plane and using Eq. (25) one obtains

$$
\begin{aligned}
& \chi_{11}(\omega)=\sum_{\lambda \in B_{u}} \frac{P_{\lambda}^{2} \cos ^{2} \phi_{\lambda}}{\omega_{T \lambda}^{2}-\omega^{2}} ; \quad \chi_{33}(\omega)=\sum_{\lambda \in B_{u}} \frac{P_{\lambda}^{2} \sin ^{2} \phi_{\lambda}}{\omega_{T \lambda}^{2}-\omega^{2}} ; \\
& \chi_{13}(\omega)=\frac{1}{2} \sum_{\lambda \in B_{u}} \frac{P_{\lambda}^{2} \sin 2 \phi_{\lambda}}{\omega_{T \lambda}^{2}-\omega^{2}} .
\end{aligned}
$$

These are contributions to the $\chi(\omega)$ tensor due to $B_{u}$ symmetry excitons. The $A_{u}$ excitons contribute only to

$$
\chi_{22}(\omega)=\sum_{\lambda \in A_{\mu}} \frac{P_{\lambda}^{2}}{\omega_{T \lambda}^{2}-\omega^{2}} .
$$

From Eq. (56), it is seen that the coefficients $k_{\alpha \alpha, \sigma \tau}(\omega)$ will have singularities at transverse exciton frequencies. The components of the tensor can now be written as

$k_{11, \sigma \tau}(\omega)=-\left\{\sum_{\lambda, \lambda^{\prime} \in B_{u}} \frac{P_{\lambda}^{2} P_{\lambda^{2}}^{2},\left(\cos ^{2} \phi_{\lambda}+\sin \phi_{\lambda} \cos \phi_{\lambda}\right)\left(\cos ^{2} \phi_{\lambda^{\prime}}+\sin \phi_{\lambda^{\prime}} \cos \phi_{\lambda^{\prime}}\right)}{\left(\omega_{\lambda}^{2}-\omega^{2}\right)\left(\omega_{\lambda}^{2},-\omega^{2}\right)}\right\}\left(R_{11, \sigma \tau}+2 R_{13, \sigma \tau}+R_{33, \sigma \tau}\right)$,

and

$k_{33, \sigma \tau}(\omega)=-\left\{\sum_{\lambda \lambda^{\prime} \in B_{u}} \frac{P_{\lambda}^{2} P_{\lambda^{\prime}}^{2}\left(\sin ^{2} \phi_{\lambda}+\sin \phi_{\lambda} \cos \phi_{\lambda}\right)\left(\sin ^{2} \phi_{\lambda^{\prime}}+\sin \phi_{\lambda^{\prime}} \cos \phi_{\lambda^{\prime}}\right)}{\left(\omega_{\lambda}^{2}-\omega^{2}\right)\left(\omega_{\lambda}^{2},-\omega^{2}\right)}\right\}\left(R_{11, \sigma \tau}+2 R_{13, \sigma \tau}+R_{33, \sigma \tau}\right)$.

These equations give insight into the microscopic origin of the elasto-optic phenomena. Namely, they show how the coefficients of the $k(\omega)$ tensor are governed by coupling between excitons.

The strain modulated reflectivity response for light normally incident on $(001)$ with polarization $E$ can be expressed as

$$
\begin{aligned}
\zeta^{\prime}(\omega)= & \frac{4 \pi}{n^{2}(\omega)-1}\left(n^{-1}(\omega) k_{11, \sigma \tau}(\omega)\right. \\
& \left.+n^{3}(\omega)\left[\epsilon^{-1}(\omega)\right]_{13} k_{33, \sigma \tau}(\omega)\right) e_{\sigma \tau} .
\end{aligned}
$$

To locate singularities of the response, calculation of frequencies corresponding to extraordinary excitons $(n \rightarrow \infty)$ and polaritons $(n \rightarrow 0)$ is necessary. 
For well-separated transitions Eqs. (53) and (56) give

$$
\epsilon_{33}^{0}(\omega)=4 \pi \frac{P_{1, B_{u}}^{2} \sin ^{2} \phi_{1}}{\omega_{T_{1}}^{2}-\omega^{2}}=0
$$

which yields the extraordinary exciton frequency measured for $\mathbf{E} \| a$ :

$$
\omega_{E_{1}}^{2}=\omega_{T_{1}}^{2}+\frac{4 \pi}{\epsilon_{33}^{0}\left(\omega_{E_{1}}\right)} P_{1, B_{u}}^{2} \sin ^{2} \phi_{1} .
$$

This expression contains the known property that the extraordinary exciton frequency exhibits directional dispersion, i.e., the frequency depends upon the angle between $\mathbf{E}$ and $\mathbf{P}$. Note that the dielectric constant $\epsilon_{33}^{0}\left(\omega_{E_{1}}\right)$, which stands for all other contributions of $B_{u}$ type to $\epsilon_{33}(\omega)$ at frequency $\omega_{E_{1}}$ except $\lambda=1$, acts as a screening constant for polarization $P_{1, B_{u}} \sin \phi_{1}$ which is a projection on the direction of the wave vector of the light $\mathbf{q} \| c^{\prime}$.

Extraordinary polariton frequencies for the assumed geometry are found from Eq. (54). When the components of the dielectric constant tensor are expressed as in Eq. (59), the polariton frequency is

$$
\omega_{P, 1}^{2}=\omega_{T, 1}^{2}+4 \pi\left[\epsilon^{0}\left(\omega_{P_{1}}\right)\right]_{q}^{-1} P_{1, B_{u}}^{2}
$$

where

$$
\begin{aligned}
{\left[\epsilon^{0}\left(\omega_{p_{1}}\right)\right]_{q}^{-1}=} & \left(\epsilon^{0}\right)_{11}^{-1} \sin ^{2} \phi_{1}-2\left(\epsilon^{0}\right)_{13}^{-1} \sin \phi_{1} \cos \phi_{1} \\
& +\left(\epsilon^{0}\right)_{33}^{-1} \cos \phi_{1}=\left(1 / n_{q}^{0}\right)^{2}
\end{aligned}
$$

is the value of the $\left[\epsilon^{0}(\omega)\right]^{-1}$ tensor at frequency $\omega_{P_{1}}$ in the direction of $q$, the wave vector of the light. Again, it gives the screening of the exciton polarization in the direction of the $q$ vector and determines directional dependence of the stopping band.

When expressing components of the dielectric tensor $\epsilon(\omega)$ such as Eq. (59) and realizing that $\epsilon^{0}(\omega)$ stands for all transitions but one, Eq. (52) becomes

$$
n^{2}(\omega)=\left[n^{0}(\omega)\right]^{2} \frac{\omega_{P_{1}}^{2}-\omega^{2}}{\omega_{E_{1}}^{2}-\omega^{2}}
$$

which can be regarded as a version of the well-known Kurosawa relation for extraordinary excitations in crystals. One has to realize that $\omega_{P_{1}}$ and $\omega_{E_{1}}$ stand for frequencies of excitations which are not purely longitudinal nor transverse as for ordinary excitons and polaritons. Equations (61) and (62) show clearly that the extraordinary polariton comes from coupling of the exciton with both components of the electric vectors $E_{1}$ and $E_{3}$ and its polarization is screened by contributions of all other excitons to the dielectric constant tensor in the direction of the wavevector of the light.

Qualitative analysis of the strain modulated reflectivity can now be made using a generalization of Eq. (63),

$$
n^{2}(\omega)=n^{2}(\infty) \prod_{\lambda} \frac{\omega_{P \lambda}^{2}-\omega^{2}}{\omega_{E \lambda}^{2}-\omega^{2}},
$$

where $\omega_{E \lambda}$ and $\omega_{P \lambda}$ are expressed by Eqs. (60) and (61), respectively. For the strain modulation, Eq. (64) gives the general expression for the modulated reflectivity response

$$
\zeta^{\prime}\left(\omega, e_{\sigma \tau}\right)=\frac{2 n(\omega)}{n^{2}(\omega)-1} \sum_{\lambda}\left[\frac{\omega_{P \lambda} \Delta \omega_{P \lambda}}{\omega_{P \lambda}^{2}-\omega^{2}}-\frac{\omega_{E \lambda} \Delta \omega_{E \lambda}}{\omega_{E \lambda}^{2}-\omega^{2}}\right],
$$

where

$$
\Delta \omega_{i, \lambda}=\frac{\partial \omega_{i, \lambda}}{\partial e_{\sigma \tau}} \cdot e_{\sigma \tau} .
$$

$\zeta^{\prime}(\omega, e)$ will exhibit singularities at both extraordinary exciton and polariton frequencies which are characteristic for a given geometry of the experiment.

The simplest case for solids will be when there is one transition responsible for optical properties over a wide range of frequency. Then the response will simply be

$$
\zeta\left(\omega, e_{\sigma \tau}\right)=\frac{1}{\omega_{P}^{2}-\omega_{E}^{2}}\left\{[n(\omega)]^{-1} \omega_{P} \Delta \omega_{P}-n(\omega) \omega_{E} \Delta \omega_{E}\right\}
$$

which, of course, behaves like $\left(1-n^{2}\right) / n$ [see Eq. (60)]. This simple equation shows an interesting feature of the $\zeta(\omega)$ signal; it is expected to be higher for smaller polariton splittings occuring in crystals of comparable elasto-optic coefficients or strain sensitivity.

It is also of interest to ascertain the relation of piezomodulated reflectivity spectra to change in the van der Waals binding energy. Since the poles of the susceptibility $X(q, \omega, e)$ [see Eq. (26)] determine the exciton frequencies for the strained crystal and the poles of $\chi(q, \omega)$ yield those for the strain free excitons, the difference

$$
\begin{aligned}
\Delta V_{d}= & \frac{\hbar}{4 \pi i N} \sum_{\mathbf{q}} \oint_{c}\left\{\ln \operatorname{det}\left|\mathrm{X}^{-1}(\mathbf{q}, \omega, \mathrm{e})\right|\right. \\
& \left.-\ln \operatorname{det}\left|\chi^{-1}(\mathbf{q}, \omega)\right|\right\} d \omega
\end{aligned}
$$

corresponds to the change in van der Waals binding energy due to the applied strain. For most molecular crystals this energy is the dominant part of the crystal cohesive energy and the change can be approximated by

$$
\Delta V_{d}=\frac{1}{2} C_{\alpha \beta, \alpha \beta} e_{\alpha \beta}^{2}
$$

which expresses the elastic energy introduced into the crystal by an applied strain $e_{\alpha \beta}$. Assuming further that spatial dispersion can be neglected and using Eq. (28),

$$
\frac{1}{2} C_{\alpha \beta, \alpha \beta} e_{\alpha \beta}^{2}=\frac{\hbar}{4 \pi i} \oint_{c} \ln \operatorname{det}\left|1+\chi(\omega) \cdot R_{\alpha \beta}(\omega) e_{\alpha \beta}\right| d \omega .
$$

Since the applied strains are always very small, it is permissible to use the expansion

$$
\ln \operatorname{det}|\mathbf{1}+\mathrm{S}|=\sum_{n=1}^{\infty} \frac{(-1)^{n+1}}{n} \operatorname{Tr}\left[\mathrm{S}^{n}\right]
$$

and truncate the series with the first term thereby obtaining

$$
\frac{1}{2} C_{\alpha \beta, \alpha \beta} e_{\alpha \beta}^{2}=\frac{h}{4 \pi i} \oint_{c} \operatorname{Tr}\left(\chi(\omega) \cdot \mathrm{R}_{\alpha \beta}(\omega) e_{\alpha \beta}\right) d \omega .
$$

For crystals of orthorhombic or higher symmetry where ordinary excitons can be measured for three polarization directions $l=1,2,3$, the equation simplifies to

$$
\frac{1}{2} C_{\alpha \beta, \alpha \beta} e_{\alpha \beta}^{2}=\frac{\hbar}{i} \sum_{T} \oint_{c} n_{l}(\omega) \zeta_{l}(\omega) d \omega
$$

An analog of Eq. (51),

$$
\zeta(\omega)=\frac{\chi_{l l}(\omega)}{4 \pi n(\omega)} R_{l l, \alpha \beta}(\omega) e_{\alpha \beta}
$$

with $\chi_{l l}(\omega)=n_{l}^{2}(\omega)-1$ has been taken as proper for ordi- 
nary excitons. Crystals of lower symmetry will have more complicated equations.

In any case, the equations show that the integrated intensity of the piezomodulated reflection spectra will provide information on the storage of elastic energy by the excitons. The equality in Eq. (71) means that the energy is assumed to be completely stored by the exciton system.

\section{CONCLUSIONS}

This paper has sought to delineate the nature of the influences determining the optical response of molecular crystals undergoing an oscillatory stress or strain. Previous experimental and theoretical work ${ }^{2-6}$ had clearly demonstrated the efficacy of such a spectroscopic technique in the study of crystals ranging from those showing quite weak to strong coupling.

The approach chosen here has been to exploit the equivalence of strain and acoustic phonons in the limit of vanishingly small values of the phonon wave vector. This provides a tie between the macroscopic strain and microscopic phonon fields. Acoustic phonons may be associated with the fluctuating induced strain.

A selective spectroscopy for the coupling of excitons mediated by the acoustic phonons results. Reflectivity measurements are of particular advantage since they allow determination of the complex dielectric function. In contrast to similar studies on metals and semiconductors where band structure calculations are necessary, the formulation of the dielectric function for molecular crystals is of much greater generality. By introduction of effective and nonlocal polarizabilities, a useful formulation of the dielectric function is achieved which is appropriate for systems such as molecular crystals where charge transfer interactions are present and which, therefore, requires consideration of short range effects. These must actually always be considered if a useful dielectric function is to be obtained.

By selection of specific experimental geometries, strains of known symmetry are generated which are responsible for excitation of a group of acoustic phonons of the same symmetry. A totally symmetric strain will couple excitons of the same symmetry while nonsymmetric strains will cause coupling of excitons of differing symmetry. The piezomodulation spectroscopy thus becomes selective by isolating various couplings of exciton states. Since the strain is tensorial, the selectivity is based upon a symmetry which is that of the lattice.

The analysis accounts for the remarkable ability of the piezomodulation spectra to reveal structure at high temperatures which is normally only observed near $4 \mathrm{~K}$. The response function for the piezomodulation shows singularities at exciton resonances for the unstrained crystal and, for properly conceived strain geometries, such singularities will be observed for solely transverse and longitudinal frequencies. The singularities in this case are shown to be of opposite sense. The magnitude of the response is inversely related to the transverse-longitudinal splitting.

Model calculations are illustrative of the results obtained in this study. Only the case I situation for pure transverse and longitudinal modes is considered. The form of the susceptibility used is that of Eq. (30) but with damping included. The modulated reflectivity for the weak coupling regime is shown in Fig. 1. A distinctive derivative-like response is obtained which is highly reminiscent of the measured modulated piezoreflection spectrum of anthracene. ${ }^{4,6}$ The convention adopted for the piezomodulation reflection response is consistent with that adopted for the experiment ${ }^{2}$ but opposite in sign to that of Eq. (51). The transverse frequencies clearly appear as maxima and the longitudinal frequencies as minima. Thus, the difference between the two extrema defines the stopping bandwidths.

The strong coupling case result is shown in Fig. 2. This was obtained by placing the oscillator resonances quite close together. The resulting curve markedly differs from that obtained in the weak coupling limit. However, the maxima still locate the transverse energies and the minima are the frequencies of the longitudinal modes. As expected, the stopping bandwidths have increased and, consistent with behavior of crystals exhibiting strong coupling, the highest energy $\mathbf{k}=0$ polariton is substantially split from the band. Indeed, the qualitative agreement with the experimental modulated piezoreflection for the strongly coupled TCNQ crystal $^{5}$ which shows quasimetallic reflection for $(010)$ is excellent. This calculation is also in agreement with the calculated modulated piezoreflection for the two level system studied previously. ${ }^{2}$ The intensities of the calculated response are not as symmetrically displaced about the zero level as was found with the weak coupling case nor are the band shapes as derivative-like in shape. This is in accord with the experimental observation which found marked difference in responses for the modulated spectrum between the weak and strong coupling regimes. ${ }^{3-5}$

The zero crossings of the modulation spectra are of particular interest since they locate frequencies at which $\epsilon_{\text {real }}=-1$. This may be generalized to $\epsilon_{\text {real }}=-\epsilon_{\text {real }}^{\prime}$ where the prime indicates that medium through which the incident light travels. The frequency range between the zero point crossing and the longitudinal mode frequency defines a region which will support surface exciton polaritons.

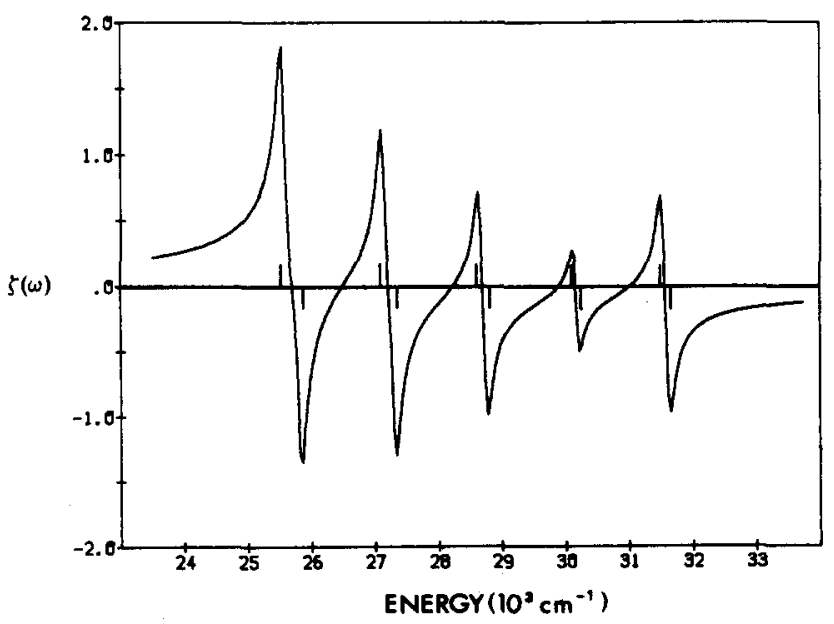

FIG. 1. Calculation of the modulated reflectivity for the weak coupling regime. A five oscillator model is used with values of $P^{2}=15.876$ (25 536), 8.864 (27 109), 5.075 (28 628), 2.231 (30 129), and 5.362 (31 500) where the frequency of the oscillator (in $\mathrm{cm}^{-1}$ ) is given in parentheses. The damping is $100 \mathrm{~cm}^{-1}$ for each. Transverse and longitudinal modes are marked by bar lines above and below the abscissa, respectively. 


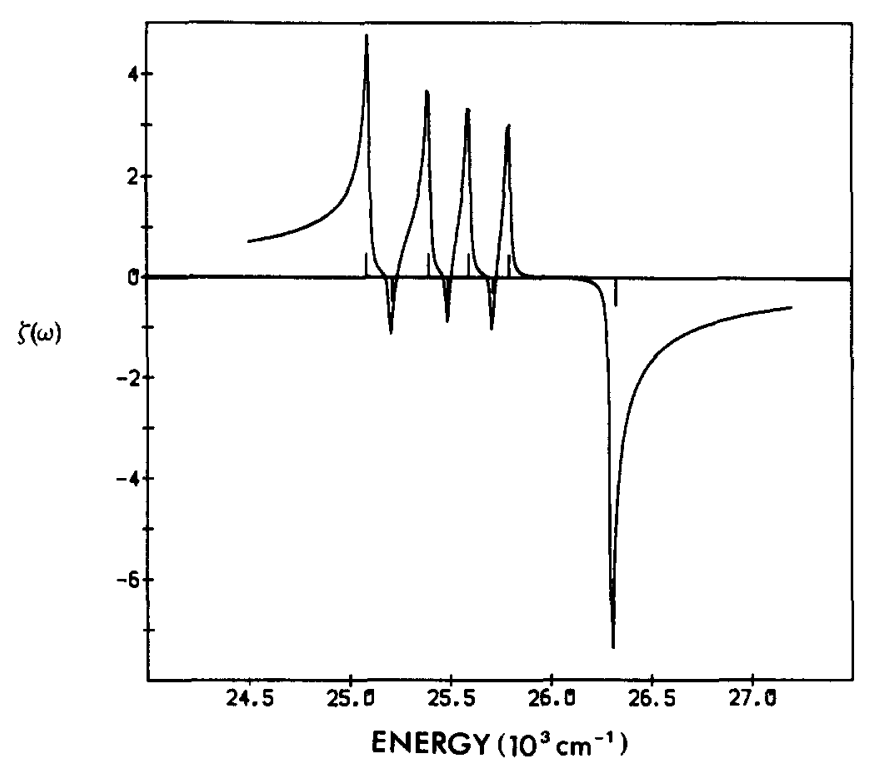

FIG. 2. Calculation of the modulated reflectivity for the strong coupling regime. A four oscillator model is used with values of $P^{2}=14.387$ (25 098), $9.995(25400), 8.9990(25600)$ and $8.000(25800)$ where the frequency of the oscillator (in $\mathrm{cm}^{-1}$ ) is given in parentheses. The damping is $15 \mathrm{~cm}^{-1}$ for each. Transverse and longitudinal modes are marked by bar lines above and below the abscissa, respectively.

The expression for the intensity of the piezomodulation provides a unique experimental approach to the study of the storage of mechanical energy by the elementary excitations of the solid. A particularly intriguing application is to the study of phase transitions and solid state reactions of molecular crystals. Those modes most involved in a particular process such as a mechanically induced phase transition should be extremely sensitive to the piezomodulation and should exhibit a large response in the modulation spectrum. Another application which is being pursued is the study of triboluminescence where the response of the piezomodulation in the infrared can be used to determine those excitations, intra- or intermolecular; most involved in the process. Such application of the piezomodulation spectroscopy may be expected to provide new experimental insights into phenomena which are related to mechanical energy interacting with the solid.

The strain is found to be independent of the driving frequency but not its amplitude in normal molecular crystals and this is consistent with the experimental observations. The strain will affect the relative orientations of the molecules in the crystal and thus piezomodulation spectroscopy provides an experimental approach to the study of internal strains. Since the amplitude of the piezomodulated reflectiv- ity can be related to the elasto-optic coefficients, the frequency dependence of these important parameters can be directly approached through experiment.

The results of this investigation demonstrate the usefulness of piezomodulation spectroscopy, and modulation spectroscopy in general, for the elucidation of the optical response of molecular crystals. This study has employed a more general analysis of the piezoreflectivity which, while completely consistent with a previous more parametrized model, ${ }^{2}$ has provided greater insight into the microscopic picture of modulation spectroscopy. This affords a straightforward approach to the interpretation of experimental results in addition to guidelines for the execution of experiments designed to exploit the selective powers of the technique.

'M. Cardona, Solid State Physics, edited by H. Ehrenreich, F. Seitz, and D. Turnbull (Academic, New York, 1969), Suppl. 11; Semiconductors and Semimetals, edited by R. K. Willardson and A. C. Beer (Academic, New York, 1972), Vol. 9.

${ }^{2} J$. Merski and C. J. Eckhardt, J. Chem. Phys. 75, 3691 (1981).

${ }^{3}$ J. Merski and C. J. Eckhardt, J. Chem. Phys. 75, 3705 (1981).

${ }^{4}$ J. Merski and C. J. Eckhardt, J. Chem. Phys. 75, 3719 (1981).

${ }^{5}$ J. Merski and C. J. Eckhardt, J. Chem. Phys. 75, 3731 (1981).

${ }^{6} \mathrm{~J}$. Merski and C. J. Eckhardt, Chem. Phys. Lett. 63, 174 (1979).

${ }^{7}$ G. D. Mahan, J. Chem. Phys. 43, 1569 (1965).

${ }^{8}$ M. R. Philpott, Adv. Chem. Phys. 23, 227 (1973); A. S. Davydov, Theory of Molecular Excitons (Plenum, New York, 1971).

${ }^{9} \mathrm{M}$. Born and K. Huang, Dynamical Theory of Crystal Lattices (Oxford University, Oxford, 1954).

${ }^{10}$ W. Hanke, Adv. Phys. 27, 287 (1978).

"S. K. Sinha, CRC Crit. Rev. Solid State Sci. 3, 273 (1973).

${ }^{12} \mathrm{G}$. Ventkataraman, L. A. Feldkamp, and V. C. Sahni, Dynamics of Perfect Crystals (MIT, Cambridge, 1975).

${ }^{13}$ R. W. Munn and T. Luty, Chem. Phys. 81, 41 (1983).

${ }^{14}$ D. F. Nelson, Electric, Optic, and Acoustic Interactions in Dielectrics (Wiley, New York, 1979).

${ }^{13}$ G. D. Mahan, J. Chem. Phys. 41, 2930 (1964).

${ }^{16}$ The conventions adopted for tensor multiplication are $\mathrm{a} \cdot \mathrm{b}=\boldsymbol{\Sigma}_{j} a_{i j} b_{j k}$ with contraction and $a_{i j} b_{k l}=\mathrm{a} \otimes \mathrm{b}$ without contraction.

${ }^{17}$ T. Luty, A. Mietzejewski, and R. W. Munn, Chem. Phys. 29, 353 (1978).

${ }^{18}$ P. J. Bounds and R. W. Munn, Chem. Phys. 24, 343 (1977).

${ }^{19}$ P. J. Bounds and R. W. Munn, Chem. Phys. 39, 165 (1979).

${ }^{20}$ E. E. Koch and A. Otto, Chem. Phys. 3, 370 (1974).

${ }^{21}$ G. D. Mahan, in NATO Advanced Study Series: Electronic Structure of Polymers and Molecular Crystals, edited by J. M. Andre and J. Ladik (Plenum, New York, 1974), p. 74.

${ }^{22}$ T. Luty and R. W. Munn. J. Phys. C 15, 4459 (1982).

${ }^{23}$ P. E. Schipper, Mol. Cryst. Liq. Cryst. 28, 401 (1974); V. I. Sugakov, in Excitons, edited by E. I. Rashba and M. D. Sturge (North-Holland, Amsterdam, 1982).

${ }^{24}$ V. M. Agranovich and M. D. Galanin, in Modern Problems in Condensed Matter Science, edited by V. M. Agranovich and A. A. Maradudin (North-Holland, Amsterdam, 1982). 